\section{Go BIG!}

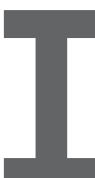

WAS FORTUNATE to enter computing in the era of site funding by the Defense Advanced Research Projects Administration (DARPA). "DARPA sites" from 1960s through the mid1990s had sustained investment of $\$ 10 \mathrm{M} /$ year (inflation adjusted). The talent and vision combined with sustained funding at scale enabled the undertaking of bold transformative ideas, such as timesharing, entire new operating systems, novel computing system architectures, new models of networking, and a raft of exciting artificial intelligence technologies-in robotics, self-driving cars, computer vision, and more. For example, I joined Arvind's Tagged-token Dataflow Computing project as an MIT graduate student. This single project, which involved a dozen graduate and undergraduates plus staff and faculty, garnered $\sim$ \$13.5M support with a run rate exceeding $\$ 4 \mathrm{M} /$ year.

Why is large-scale funding important? It enables examination of larger questions that cross problem spaces, systems, abstractions, even fields. Yes, we have open source software. Yes, we can build on large-scale frameworks and software systems. Yes, we can compose services and leverage the cloud. But it is important to recognize that leverage of such infrastructures often means that rethinking or reimagining them is beyond the scope of inquiry. I believe the need for such large investments has never been greater. We are seeing:

- Transformative change in architecture, operating systems, programming languages, and applications. New large-scale geographic and distributed structures-datacenters, edge, home, undersea, orbital systems and soon outer space.
- New applications from computing, data, intelligence, and trusted algorithmic execution (for example, blockchain) melding with a variety of fields - law, business, in new ways with accelerating benefit to society.

- Information technology in the body politic, journalism, and social fabric writ large- "fake news," "social network addiction and depression," and "societal manipulation."

In the past decade, we have seen extraordinary new capabilities. GPU computing has enabled new levels of energy efficient, high performance, but only with radical software change (and of course architecture). The reemergence of neuromorphic computing (including deep learning) delivered new artificial intelligence capabilities to a broad array of applications. And visionaries suggest many more eruptions are coming. ${ }^{1}$

Numerous National Academy and committee reports have called for research "big bets" based on need and opportunity. And in late 2007, during my service on the NSF CISE Advisory Committee, then-CISE AD Jeannette Wing initiated the NSF CISE Expeditions program to create "big bets."2 A bright spot! But with a much smaller investment than prior programs, funding only 1.5 projects per year (each $\sim \$ 10 \mathrm{M} /$ five years). Expeditions are terrific projects, but typically split over several sites, diluting infrastructure, capability, and perspective. For example, a 2018 Expedition Award, "EPiQC: Enabling Practical-Scale Quantum Computation," led by my colleague Fred Chong at the University of Chicago, includes five institutions.

Given industrial-scale R\&D, does computing need large-scale government research investments? Yes! Academic research has fundamental advantages for society, including education and broad idea and technology dissemination; openness to a wide range of possibilities and directions (not just "our business position"); vetted and publicly examined rigorously from a scientific point of view (for example, bias in algorithms, security architectures, privacy and exploitation); and vetted and publicly examined from a broad societal perspective (for example, social media, addictive technology).

So what would I propose?

Perhaps a new program. Perhaps a doubling, and doubling again of the Expeditions program. Projects of twice the scale (\$20M over five years) with mechanisms to ensure they are concentrated at no more than two institutions. And doubling the number of such efforts to three new starts every year. Too expensive? Such a program would be less than $1 / 5$ of $1 \%$ of the NIH's annual budget, and $1 / 100$ of $1 \%$ of the U.S. Department of Defense budget.

Perhaps if we want to increase global economic growth beyond what economists call "structural limits," we need more disruptive computing advances. Carpe Diem!

Andrew A. Chien, EDITOR-IN-CHIEF

Andrew A. Chien is the William Eckhardt Distinguished Service Professor in the Department of Computer Science at the University of Chicago, Director of the CERES Center for Unstoppable Computing, and a Senior Scientist at Argonne National Laboratory.

\section{References}

1. Conte, T.M., DeBenedictis, E.P., Gargini, P.A., and Track, E. Rebooting computing: The road ahead. Computer 50, 1 (2017), 20-29.

2. NSF Expeditions in Computing Program; www.nsf.gov/ 